\title{
Evaluating Sensitivity of ipsogen BCR-ABL1 Mbcr IS-MMR DX Kit for Scoring Molecular Response
}

\author{
Akiko Mita ${ }^{1}$, Koichi Miyamura ${ }^{2}$, Masayuki Hino ${ }^{3}$, Kyoko Watakabe ${ }^{2}$, Keita Takahashi ${ }^{4}$, Michiko Yoshimoto ${ }^{4}$ and Naoto Takahashi $^{{ }^{*}}$ \\ ${ }^{1}$ Department of Hematology, Nephrology, and Rheumatology, Akita University, Japan \\ ${ }^{2}$ Depertment of Hematology, Japanese Red Cross Nagoya First Hospital, Japan \\ ${ }^{3}$ Department of Hematology, Graduate School of Medicine, Osaka City University, Japan \\ ${ }^{4}$ Sysmex Corporation, Japan
}

*Corresponding author: Naoto Takahashi, Department of Hematology, Nephrology, and Rheumatology, Akita University, 1-1-1 Hondo, Akita City, Akita-010-8543, Japan, Tel: +81-18-884-6115; Fax: +81-18-836-2613; E-mail: naotot@doc.med.akita-u.ac.jp

Received date: Sep 01, 2015, Accepted date: Oct 05, 2015, Publication date: Oct 10, 2015

Copyright: $\odot 2015$ Mita A, et al. This is an open-access article distributed under the terms of the Creative Commons Attribution License, which permits unrestricted use, distribution, and reproduction in any medium, provided the original author and source are credited.

\begin{abstract}
Objective: Tyrosine kinase inhibitors (TKIs) are the standard of care for patients with chronic myeloid leukemia (CML). Recent studies demonstrated that some patients with CML could sustain remission under discontinuation of TKIs. The clinical decision to discontinue such therapy depends on the patient's molecular response (MR), derived from the international scale of BCR-ABL1 transcripts per internal control gene transcripts (BCR-ABL1IS), with MR4.5 (i.e., BCR-ABL1' $\leq 0.0032 \%$ ) commonly used as a threshold for discontinuation of TKI. However, it remains controversial whether all methods for determining BCR-ABL1/S have sufficient sensitivity for measuring MR4.5.
\end{abstract}

Methods: Probit analysis with reference to CLSI EP-17-A2 was used for the kit-specific limit of detection (LoD) of BCR-ABL1 transcripts measured by the ipsogen BCR-ABL1 Mbcr IS-MMR DX Kit. In total, 50 samples of peripheral blood (PB) were collected from CML patients across three sites, with each 21-ml sample split into 7- and 14-ml volumes.

Results: The kit-specific LoD was determined as 3 copies/assay. The sample measurements of $94 \%$ (7-ml PB group) and 96\% (14-ml PB group) showed over 94000 copies/assay of ABL1 transcripts, which is the level required to score MR4.5 considering kit-specific LoD of 3 copies/assay.

Conclusion: This result demonstrated that the ipsogen BCR-ABL1 Mbcr IS-MMR DX Kit possesses sufficient sensitivity for stable scoring at MR4.5, with strict conditions set for avoiding false-negative results.

Keywords: Chronic myeloid leukemia; TKI discontinuation; BCRABL1; RT-qPCR; Sensitivity

\section{Background}

Treatment with tyrosine kinase inhibitors (TKIs) dramatically improves survival in chronic myeloid leukemia (CML) patients and is thus the standard of care agent for CML [1]. The monitoring of CML therapeutics is based on BCR-ABL1 ${ }^{\mathrm{IS}}$ levels measured by reverse transcription quantitative PCR (RT-qPCR). The BCR-ABL $1^{\mathrm{IS}}$ was defined as the international scale converted from measurements of BCR-ABL1 transcripts relative to those of an internal control gene, most commonly ABL1, using a lab-specific conversion factor (CF) or commercial kits that can report BCR-ABL1 ${ }^{\mathrm{IS}}[2]$.

Recently, the STop IMatinib (STIM) study showed that 39\% of imatinib-discontinued CML patients who had shown no detectable BCR-ABL1 transcripts for at least two years before discontinuation were able to sustain treatment-free remission [3]. A number of prospective and retrospective studies of TKI discontinuation followed and reported findings similar to those arising from the STIM study [4-6]. However, these trials used a variety of molecular response (MR) definitions to screen for eligibility for TKI discontinuation, which leads to difficulty in comparing these studies. In the meantime, a definition of MR was proposed by Cross et al. [2] as "MR4 equals either (i) detectable disease with $0.01 \%$ BCR-ABL $1^{\mathrm{IS}}$ or (ii) undetectable disease in cDNA with 10000 ABL1 transcripts; MR4.5 equals either (i) detectable disease with $0.0032 \%$ BCR-ABL $1^{\mathrm{IS}}$ or (ii) undetectable disease in cDNA with 32000 ABL1 transcripts". The same group also proposed detailed laboratory recommendation that lab-specific limit of detection (LoD) of BCR-ABL1 transcripts should be used to calculate BCR-ABL1IS in case of a positive result lower than the LoD. The Minimum Information for Publication of Quantitative Real-Time PCR Experiments (MIQE) guideline states that the most sensitive LoD of RT-qPCR theoretically possible is 3 copies/assay, assuming a Poisson distribution [7]. Therefore, if using a method whose LoD of BCRABL1 transcripts is 3 copies/assay, scoring MR4.5 requires at least 94 000 ABL1 transcripts per PCR in cases of undetectable disease, in case the $\mathrm{CF}$ is 1 .

The ipsogen BCR-ABL1 Mbcr IS-MMR DX Kit can report BCR$A B L 11^{I S}$ derived from RT-qPCR measurements using a calibrator whose value is assigned according to WHO 1st material [8]. According to Miyamura et al. [9] this kit possesses high accuracy and low inter-lab variability; however, the manufacturer does not supply a sensitivity value for scoring MR4.5. 
Here, we evaluated the distribution of ABL1 transcripts using the ipsogen BCR-ABL1 Mbcr IS-MMR DX Kit to clarify whether this kit can score MR4.5 stably with consideration of the kit-specific LoD.

\section{Materials and Methods}

\section{Determination of kit-specific LoD}

The LoD of BCR-ABL1 transcripts measured by the ipsogen BCRABL1 Mbcr IS-MMR DX Kit was derived from the probit analysis with reference to CLSI EP-17-A2. Six serial-diluted samples were prepared by mixing RNA from BCR-ABL1-negative cell lines with RNA from the BCR-ABL1-positive cell line (K562) to be less than 10 copies per assay tube.

\section{Patients and study design}

$\mathrm{Ph}$-positive CML patients were enrolled in this study and $21 \mathrm{ml}$ of peripheral blood $(\mathrm{PB})$ was collected from each patient using EDTA tubes. Each PB sample was divided into 7- and 14-ml volumes, and these were independently subjected to RNA extraction and measurement using an ipsogen BCR-ABL1 Mbcr IS-MMR DX Kit. This study was conducted in accordance with the Helsinki Declaration and approved by the appropriate institutional review board. All participants gave written informed consent.

\section{Extraction of RNA and measurement of BCR-ABL1 ${ }^{\text {IS }}$}

RNA was extracted from the PB buffy coat within 24 hours after blood collection using the RNeasy Mini Kit (QIAGEN, Germany) with $40 \mu \mathrm{l}$ of elution buffer from both 7- and 14-ml PB samples. Then, the BCR-ABL1 and ABL1 transcripts in $10 \mu$ of RNA without dilution were measured using the RT-qPCR-based ipsogen BCR-ABL1 Mbcr IS-MMR DX Kit (Manufactured by QIAGEN Marseille and distributed by Sysmex in Japan) using an Applied Biosystems 7500 Fast Real-Time PCR System (Applied Biosystems, Foster City, CA), and additional measurement was performed with diluted RNA (100 ng/ $\mu \mathrm{l})$. The BCRABL1 and ABL1 transcripts were calibrated using 10 to 1000000 plasmid calibrators and 1000 to 1000000 plasmid calibrators, respectively, and measured in parallel with RNA of the kit-included ISMMR Calibrator (IS-Cal), whose value had been assigned according to WHO 1 st material. BCR-ABL1 ${ }^{\mathrm{IS}}$ was calculated as shown in Figure 1.

$$
\begin{aligned}
& |S-C a|(\%)=\frac{B C R-A B L 1 \text { transcripts of } I S-C a \mid}{A B L 1 \text { transcripts of } I S-C a \mid} \times 100 \\
& \text { IS conversion factor }=\frac{\text { Assigned value of IS-CaI (IS\%) }}{\downarrow \quad I S \text {-CaI (\%) }} \\
& \text { Sample }(I S \%)=\frac{B C R-A B L 1 \text { transcripts of sample }}{A B L \text { transcripts of sample }} \times 100 \times 1 S \text { conversion factor }
\end{aligned}
$$

Figure 1: Formula for IS conversion of the ipsogen BCR-ABL1 Mbcr IS-MMR DX Kit results.IS\%: BCR-ABL1 ${ }^{\mathrm{IS}}$.

\section{Statistical analysis}

Statistical analysis was carried out using R (ver. 3.02). Kit-specific LoDs were determined by probit analysis (95\% detection rate) with reference to CLSI EP-17-A2. ABL1 transcripts required for scoring MR were calculated using the kit's LoD of 3 copies/assay and the IS conversion factor of 1 , which is the median of all measurements in this study as shown in the Results section (e.g. 3/[Required ABL1 transcripts $\times 1 \leq 0.0032 \%$ used in calculation for scoring MR4.5), which was a criterion value for calculating whether the sample population reached sufficient sensitivity for MR scoring. Differences between the 7-ml and 14-ml PB samples in RNA concentration or ABL1 transcripts were analyzed by the Wilcoxon signed-rank test. Correlation was analyzed based on Pearson's correlation coefficient and regression analysis was performed by the least squares method.

\section{Results}

The LoD of BCR-ABL1 transcripts measured using the ipsogen BCR-ABL1 Mbcr IS-MMR DX Kit was determined by probit analysis. A boxplot of all measurements for six samples is shown in Figure 2A. One measurement of sample L was removed as an outlier determined to be outside the $75^{\text {th }}$ percentile plus 3 IQR. Summary of BCR-ABL1 transcripts including the hit rate is provided in Table 1 . To determine the LoD, the hit rate was fitted with a probit regression of the expected logarithmic BCR-ABL1 transcripts. After this fitting, the LoD was the BCR-ABL1 transcript corresponding to a $95 \%$ hit rate. In the density function of a Gaussian distribution, the $95^{\text {th }}$ percentile is 1.645 . Thus, the LoD is derived using the following formula,

$\mathrm{LoD}=10^{((1.645-\text { Probit intercept }) /(\text { Probit slope }))}$

Using the probit analysis, the LoD of BCR-ABL1 transcripts were determined as 3 copies/assay rounded up from 2.0921 copies/assay (Figure 2B).

Fifty adult patients diagnosed with $\mathrm{Ph}$-positive $\mathrm{CML}$ were enrolled from three sites. Table 2 presents the demographics including BCR$\mathrm{ABL}^{\mathrm{IS}}$ for all patients measured in this study.

We collected $21 \mathrm{ml}$ of $\mathrm{PB}$ from all participants and RNA was extracted from component 7- and 14-ml samples using $40 \mu \mathrm{l}$ elution buffer (median [min-max] RNA concentrations of 278 [98-738] ng/ $\mu \mathrm{l}$ and 385 [145-704] $\mathrm{ng} / \mu \mathrm{l}$, respectively; $\mathrm{P}<0.000)$. A ratio of medians derived from the two groups was 1.4.

Nine assay batches were tested using the ipsogen BCR-ABL1 Mbcr IS-MMR DX Kit, and the median IS-conversion factor derived for each batch is 1.02, as shown in Figure 3.

Figure 4 represents the distribution of ABL1 transcripts obtained from each volume of $\mathrm{PB}(7 \mathrm{ml}$ and $14 \mathrm{ml})$ across the patients; the medians [min-max] were 174601 [82 183-420 826] copies/assay and 190010 [58 959-335 736] copies/assay, respectively ( $\mathrm{P}=0.004)$, with a ratio of medians derived from the two groups of 1.1.

To investigate if the ipsogen BCR-ABL1 Mbcr IS-MMR DX Kit possesses sufficient sensitivity to provide stable MR scoring, we analyzed the proportion of samples containing the required ABL1 transcript levels for scoring each MR (Table 3), finding that $94 \%$ and $96 \%$ of the $7 \mathrm{ml}$ and $14 \mathrm{ml}$ samples, respectively, contained more than 94000 copies/assay of ABL1 transcripts, as required for a score of MR4.5 under the condition of kit-specific LoD of 3 copies/assay and an IS-conversion factor of 1.0, which is the median of all assay batches in this study.

To investigate if ABL1 transcript level was associated with RNA concentration in this study, we performed correlation analysis, and found significant correlations for both the 7-ml $\mathrm{PB}$ group and 14-ml $\mathrm{PB}$ group as expected $[\mathrm{r}=0.875 \quad(\mathrm{P}<0.001), \quad \mathrm{r}=0.694 \quad(\mathrm{P}<0.001)$, 
Citation: $\quad$ Mita A, Miyamura K, Hino M, Watakabe K, Takahashi K, et al. (2015) Evaluating Sensitivity of ipsogen BCR-ABL1 Mbcr IS-MMR DX Kit for Scoring Molecular Response. J Blood Disord Transfus 6: 314. doi:10.4172/2155-9864.1000314

Page 3 of 5

respectively] (Figure 5). As shown in Figure 6, correlation and regression coefficients between $\mathrm{BCR}-\mathrm{ABL} 1^{\mathrm{IS}}$ from undiluted and diluted samples were close to 1 in both the $7-\mathrm{ml} \mathrm{PB}$ and $14-\mathrm{ml} \mathrm{PB}$ groups. This result indicates that BCR-ABL1IS is not affected by RNA concentration at least between $98-738 \mathrm{ng} / \mu \mathrm{l}$ in the $7-\mathrm{ml} \mathrm{PB}$ group and $145-704 \mathrm{ng} / \mu \mathrm{l}$ in the 14-ml PB group.

\begin{tabular}{|l|l|l|l|}
\hline Sample name & $\mathbf{N}$ & Expected value (copies/assay) & Hit rate (\%) \\
\hline G & 20 & 7.22 & 100 \\
\hline H & 20 & 4.81 & 100 \\
\hline I & 20 & 3.21 & 100 \\
\hline J & 20 & 2.14 & 95 \\
\hline K & 20 & 1.43 & 80 \\
\hline L & 19 & 0.95 & 63 \\
\hline
\end{tabular}

Table 1: Summary of BCR-ABL1 transcripts after outlier removal.
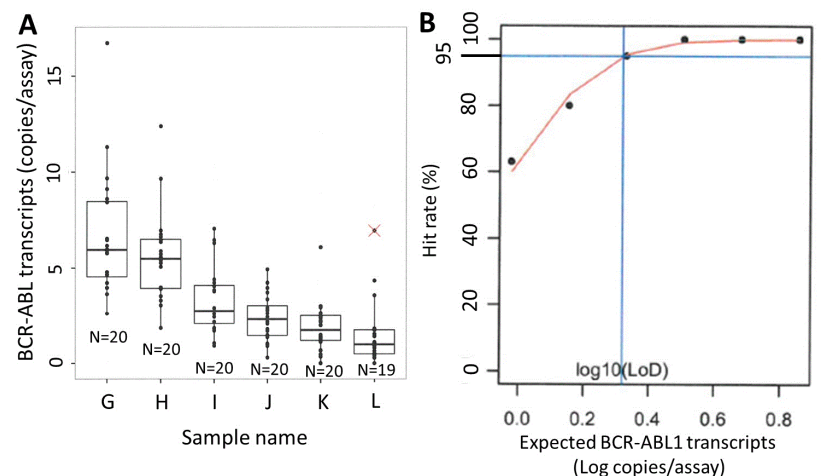

Figure 2: LoD determination of BCR-ABL1 transcripts measured by the ipsogen BCR-ABL1 Mbcr IS-MMR DX Kit. (A) Box plot of BCR-ABL1 transcripts obtained from 6 serial dilution samples. The measurements associated with the cross lie outside the 75th percentile plus 3 IQR. (B) Probit regression curve obtained from 6 serial dilution samples.

\begin{tabular}{|c|c|}
\hline & Data \\
\hline Age (median years [min-max]) & $56.45[21-82]$ \\
\hline \multicolumn{2}{|l|}{ Sex } \\
\hline Male, $\mathrm{n}(\%)$ & $36(60)$ \\
\hline Female, $n(\%)$ & $24(40)$ \\
\hline WBC count $(\text { median } / \mu \mathrm{l}[\min -\mathrm{max}])^{*} 1$ & 6000 [2400-12500] \\
\hline BCR-ABL IS (median\% [min-max]) 2 & $0.0035[0-19.4738]$ \\
\hline
\end{tabular}

Table 2: Patient demographics.

\begin{tabular}{|l|l|l|}
\hline & $\mathbf{7 ~} \mathbf{~ m l ~ ( N = 5 0 )}$ & $\mathbf{1 4} \mathbf{~ m l ~ ( N = 5 0 ) ~}$ \\
\hline MR4, n(\%) & $50(100)$ & $50(100)$ \\
\hline MR4.5, $n(\%)^{*}$ & $47(94)$ & $48(96)$ \\
\hline MR5, $\mathrm{n}(\%)^{*}$ & $2(4)$ & $5(10)$ \\
\hline $\begin{array}{l}\text { MR: Molecular Response } \\
\text { "Number of samples containing ABL1 transcripts required for scoring each MR }\end{array}$
\end{tabular}

Table 3: Proportion of samples possessing sensitivity for scoring MR.

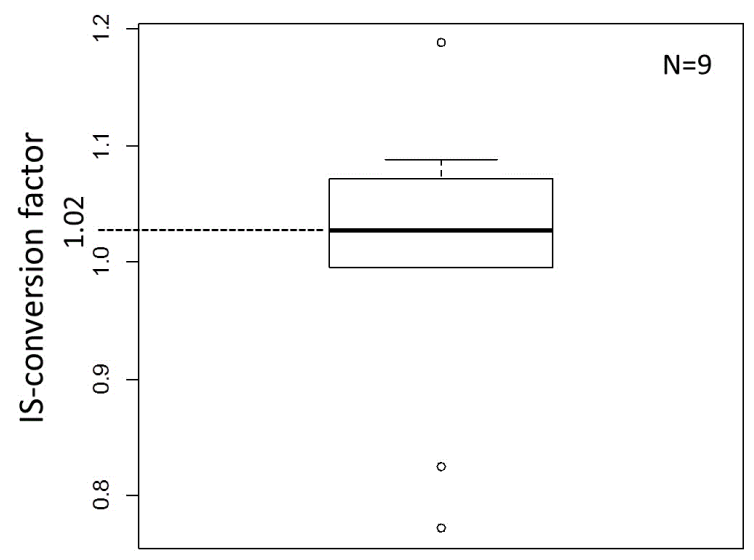

Figure 3: Distribution of IS-conversion factors. Box plot of ISconversion factor derived from 9 assay batches in this study.
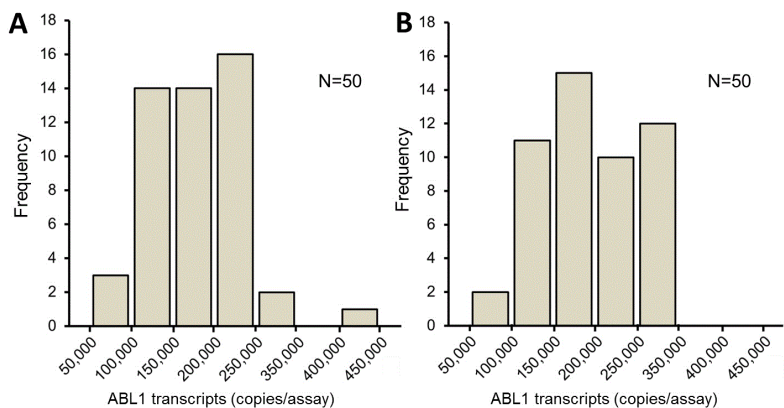

Figure 4: Distribution of ABL1 transcripts. Distribution of ABL1 transcripts measured by the ipsogen BCR-ABL1 Mbcr IS-MMR DX Kit with 7-ml PB (A) and 14-ml PB (B) samples. 


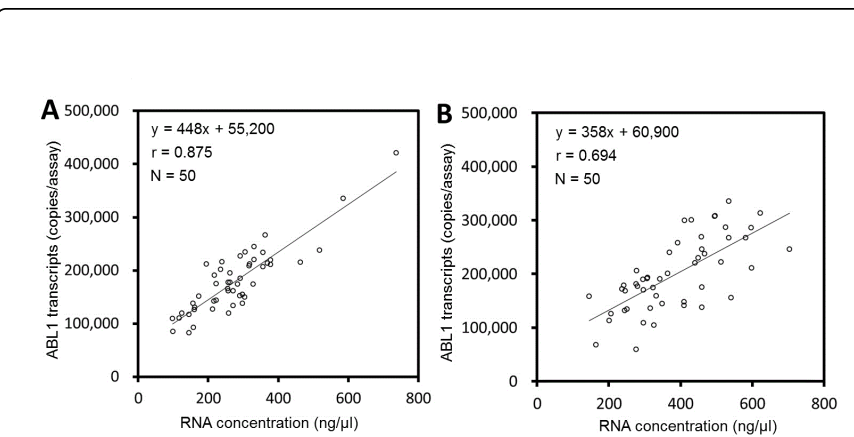

Figure 5: Correlation between ABL1 transcripts and RNA concentration. Correlation analyzed between ABL1 transcripts and RNA concentration obtained from the 7-ml PB (A) and 14-ml PB (B) samples.
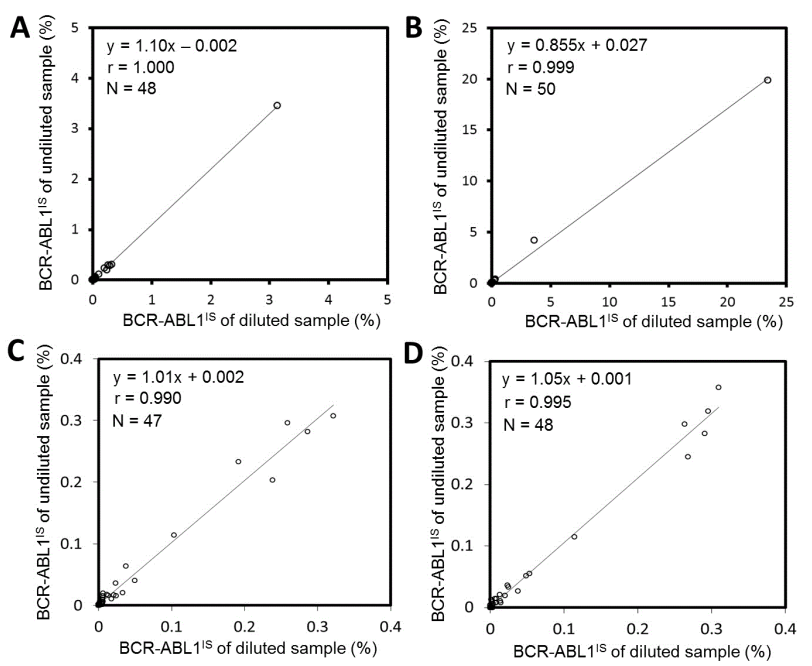

Figure 6: Correlation between $\mathrm{BCR}-\mathrm{ABL} 1^{\mathrm{IS}}$ of diluted and undiluted samples. Correlation analyzed between BCR-ABL $1^{\mathrm{IS}}$ of diluted (100 $\mathrm{ng} / \mu \mathrm{l})$ and undiluted samples obtained from the 7-ml PB sample. A: Samples in entire measurement range, C: Samples up to $1 \%$ ) and 14-ml PB sample. B: Samples in entire measurement range, C: Samples up to $1 \%)$.

\section{Discussion}

Scoring of a patient's MR based on measurement of BCR-ABL1 ${ }^{\mathrm{IS}}$ is essential for the management of CML because it is used to decide both changes of treatment in routine practice and cessation of treatment in clinical studies [5]. Especially for TKI discontinuation, an extremely low level of measurement including undetectable BCR-ABL1 transcripts is required for MR scoring. Although quantitation of BCRABL1 transcripts has been standardized by the contribution of IS standardization carried out after the IRIS trial [10], standardization of scoring MR in cases of extremely low measurements including undetectable BCR-ABL1 transcripts has not been completed. In this study, we evaluated the sensitivity of an ipsogen BCR-ABL1 Mbcr IS-
MMR DX Kit to investigate ABL1 transcript measurements with consideration of the kit-specific LoD.

We investigated the kit sensitivity in two groups, the 7-ml and 14-ml groups. The sample measurements of $94 \%$ (7 ml PB group) and $96 \%$ (14 ml PB group) showed over 94000 copies/assay of ABL1 transcripts, which satisfied the sensitivity for scoring MR4.5 based on the kitspecific LoD of 3 copies/assay and IS conversion factor of 1 . Although RNA concentration and ABL1 transcripts for the 14-ml PB volumes were twice as much as that of the $7-\mathrm{ml}$ samples theoretically, the ratios of median derived from RNA concentration and ABL1 transcripts between the groups were 1.4 fold and 1.1 fold, respectively. Saturation of the RNA extraction column and a decrease in efficiency of reverse transcription could cause such a difference from the theoretical value; however, a specific cause was not elucidated in this study. Considering patient burden, $7 \mathrm{ml}$ should be sufficient for the ipsogen BCR-ABL1 Mbcr IS-MMR DX Kit in scoring MR4.5. Furthermore, less than $7 \mathrm{ml}$ might be appropriate with consideration of patient burden, although such a question could not be addressed in this study.

To investigate possible factors for predicting ABL1 transcripts before blood collection, we tested for a correlation between white blood cell (WBC) count of enrolled patients before and after 1 month from blood correction and ABL1 transcript level measurements, with no significant correlations found in either group $[\mathrm{r}=0.179(\mathrm{P}=0.215)$, $\mathrm{r}=-0.017(\mathrm{P}=0.905)$, respectively]. This result indicates not only that it is difficult to select the best volume of $\mathrm{PB}$ to collect according to WBC count, but also that an option of $14 \mathrm{ml} \mathrm{PB}$ sampling is not required in routine practice.

It was confirmed that BCR-ABL1 ${ }^{\mathrm{IS}}$ measured with the ipsogen BCRABL1 Mbcr IS-MMR DX Kit was not affected by RNA concentration between $98-738 \mathrm{ng} / \mu \mathrm{l}$ in the $7-\mathrm{ml}$ sample and $145-704$ in the $14-\mathrm{ml}$ volume. These data take into account that the extracted RNA without dilution should be subjected to measurement using the ipsogen BCRABL1 Mbcr IS-MMR DX Kit for stably scoring MR4.5 when RNA concentration is within $10-1000 \mathrm{ng} / \mu \mathrm{l}$.

Cross et al. [11] recently reported that the accuracy and precision by which MR can be scored critically depends on the LoD of BCR-ABL1 transcripts being maximized, and recommended that the LoD value should be used for calculating MR if BCR-ABL1 transcripts are lower than the LoD "without undetectable disease". Since LoD is defined as a lower limit by which to distinguish a positive and undetectable result, the LoD value should be considered more useful for calculating required transcripts of the internal control gene "in cases of undetectable diseases", thus avoiding false-negative results.

In conclusion, this study confirmed that the ipsogen BCR-ABL1 Mbcr IS-MMR DX Kit possesses sufficient sensitivity for stably scoring MR4.5 according to the strictest conditions for avoiding false-negative results.

\section{Acknowledgments}

This study was conducted under the sponsorship of Sysmex Corporation. We thank members of QIAGEN Marseille SA for handling the analytical studies using the ipsogen BCR-ABL1 Mbcr ISMMR DX Kit.

\section{Conflict of Interest}

This study was conducted under the sponsorship of Sysmex Corporation. 
Citation: $\quad$ Mita A, Miyamura K, Hino M, Watakabe K, Takahashi K, et al. (2015) Evaluating Sensitivity of ipsogen BCR-ABL1 Mbcr IS-MMR DX Kit for Scoring Molecular Response. J Blood Disord Transfus 6: 314. doi:10.4172/2155-9864.1000314

Page 5 of 5

N.T., H. M., W. K. and K. M. have received research funding from Sysmex Corporation. K. T. and Y. M. are employees of Sysmex Corporation.

\section{References}

1. Druker BJ, Guilhot F, O'Brien SG, Gathmann I, Kantarjian H, et al. (2006) Five-year follow-up of patients receiving imatinib for chronic myeloid leukemia. N Engl J Med 355: 2408-2417.

2. Cross NC, White HE, Müller MC, Saglio G, Hochhaus A (2012) Standardized definitions of molecular response in chronic myeloid leukemia. Leukemia 26: 2172-2175.

3. Mahon FX, Rea D, Guilhot J, Guilhot F, Huguet F, et al. (2010) Discontinuation of imatinib in patients with chronic myeloid leukaemia who have maintained complete molecular remission for at least 2 years: the prospective, multicentre Stop Imatinib (STIM) trial. Lancet Oncol 11: 1029-1035.

4. Takahashi N, Kyo T, Maeda Y, Sugihara T, Usuki K, et al. (2012) Discontinuation of imatinib in Japanese patients with chronic myeloid leukemia. Haematologica 97: 903-906.

5. Ross DM, Branford S, Seymour JF, Schwarer AP, Arthur C, et al. (2013) Safety and efficacy of imatinib cessation for CML patients with stable undetectable minimal residual disease: results from the TWISTER study. Blood 122: 515-522.
6. Yhim HY, Lee NR, Song EK, Yim CY, Jeon SY, et al. (2012) Imatinib mesylate discontinuation in patients with chronic myeloid leukemia who have received front-line imatinib mesylate therapy and achieved complete molecular response. Leuk Res 36: 689-693.

7. Bustin SA, Benes V, Garson JA, Hellemans J, Huggett J, et al. (2009) The MIQE guidelines: minimum information for publication of quantitative real-time PCR experiments. Clin Chem 55: 611-622.

8. White HE, Matejtschuk P, Rigsby P, Gabert J, Lin F, et al. (2010) Establishment of the first World Health Organization International Genetic Reference Panel for quantitation of BCR-ABL mRNA. Blood 116: e111-117.

9. Miyamura K, Okamoto S, Usui N, Hino M, Akashi K, et al. (2014) Evaluation of clinical performance of the major BCR-ABL mRNA detection kit which enables conversion to international standard scale using the reference material calibrator. Rinsho Ketsueki 55: 534-540.

10. Hughes T, Deininger M, Hochhaus A, Branford S, Radich J, et al. (2006) Monitoring CML patients responding to treatment with tyrosine kinase inhibitors: review and recommendations for harmonizing current methodology for detecting BCR-ABL transcripts and kinase domain mutations and for expressing results. Blood 108: 28-37.

11. Cross NC, White HE, Colomer D, Ehrencrona H, Foroni L, et al. (2015) Laboratory recommendations for scoring deep molecular responses following treatment for chronic myeloid leukemia. Leukemia 29: 999-1003. 